\title{
Analyzing (initial) biotransformation reactions as an organizing principle to unravel the extent of trace organic chemical biotransformation in biofiltration systems
}

\author{
Uwe Hübner ${ }^{a} *^{+}$, David Wolff $^{b+}$, Stefan Achermann ${ }^{c}$, Jörg E. Drewes ${ }^{a}$, Arne Wick ${ }^{b}$, \\ Kathrin Fenner ${ }^{c}$

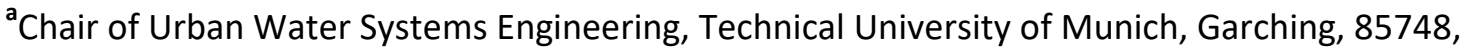 \\ Germany \\ ${ }^{b}$ Federal Institute of Hydrology, Koblenz, 56068, Germany \\ 'Eawag, Swiss Federal Institute of Aquatic Science and Technology, Dübendorf, 8600, Switzerland
}

SI1 Measured concentrations of trace organic chemicals in column experiments Measured concentrations of trace organic chemicals in batch biodegradation tests Measured concentrations of trace organic chemicals in batch sorption tests Determined rate constants from batch biotransformation experiments Gene copy numbers in the sands originating from the different columns Removal of TOrCs in column and batch experiments Evaluation of plausible initial transformation reaction based on literature data and pathway prediction 


\begin{tabular}{|c|c|c|c|c|c|c|c|c|c|c|}
\hline Compound & Laboratory & LOQ & B01_Inf & B01 & B02 & b1_Inf & b1 & b2 & b3 & b4 \\
\hline 5-Methyl-Benzotriazol & Eawag & 50 & $0935.5 \pm 200.3$ & $791.7 \pm 137.8$ & $775.8 \pm 167.1$ & $789.5 \pm 105.4$ & $670.3 \pm 87$ & $725.8 \pm 96.5$ & $760.1 \pm 77.7$ & $846.1 \pm 63.6$ \\
\hline Acesulfame & Eawag & 100 & $04398.8 \pm 821$ & $162.7 \pm 21.2$ & $155.8 \pm 23.2$ & $120.5 \pm 20.5$ & $130.7 \pm 23.7$ & $162 \pm 0$ & $138 \pm 33$ & $135.3 \pm 25$ \\
\hline Acetamiprid & Eawag & 50 & $03459.6 \pm 557.6$ & $2135.1 \pm 176.6$ & $2071.3 \pm 137.1$ & $1966.8 \pm 178.9$ & $60.7 \pm 0$ & $56 \pm 0$ & $<\mathrm{LOQ}$ & $<\mathrm{LOQ}$ \\
\hline Acyclovir & BfG & & $034.5 \pm 6.6$ & $<\mathrm{LOQ}$ & $<\mathrm{LOQ}$ & $<$ LOQ & $<\mathrm{LOQ}$ & $<$ LOQ & $<L O Q$ & $<L O Q$ \\
\hline Alachlor & Eawag & 200 & $0540.4 \pm 66$ & $411.1 \pm 29.2$ & $379.1 \pm 12.6$ & $261 \pm 37.6$ & $<L O Q$ & $<\mathrm{LOQ}$ & $<L O Q$ & $<L O Q$ \\
\hline Amisulpride & BfG & & $21228.3 \pm 656.2$ & $889.3 \pm 730.6$ & $624.2 \pm 409.4$ & $719.3 \pm 658.3$ & $5.2 \pm 1.9$ & $6 \pm 0$ & $<\mathrm{LOQ}$ & $<\mathrm{LOQ}$ \\
\hline Atenolol & $\mathrm{BfG}$ & 20 & $02594.1 \pm 757.5$ & $472.3 \pm 214.1$ & $302.1 \pm 175.3$ & $268.1 \pm 156.6$ & $<$ LOQ & $<L O Q$ & $<$ LOQ & $<$ LOQ \\
\hline Azithromycin & $\mathrm{BfG}$ & 100 & $0163.4 \pm 0$ & $<$ LOQ & $<$ LOQ & $<$ LOQ & $<$ LOQ & $<\mathrm{LOQ}$ & $<L O Q$ & $<$ LOQ \\
\hline Azoxystrobin & Eawag & 50 & $0871.5 \pm 132.9$ & $731.2 \pm 79$ & $773.5 \pm 126.7$ & $277 \pm 96.2$ & $163.3 \pm 47.2$ & $105.8 \pm 30.4$ & $78 \pm 0$ & $<\mathrm{LOQ}$ \\
\hline Benzotriazole & BfG & & $06724.2 \pm 1286.3$ & $34068.9 \pm 1031.1$ & $13982.2 \pm 1053$ & $2898.9 \pm 1857.3$ & $551.8 \pm 42.8$ & $734.3 \pm 81.3$ & $723.8 \pm 71.6$ & $651.6 \pm 199.4$ \\
\hline Bezafibrate & $\mathrm{BfG}$ & & .0 $2290.5 \pm 499.3$ & $479 \pm 103$ & $198.9 \pm 73.3$ & $129.2 \pm 73.3$ & $<L O Q$ & $<L O Q$ & $<$ LOQ & $<$ LOQ \\
\hline Bromoxynil & Eawag & & $03150.2 \pm 587.3$ & $2885 \pm 138.2$ & $2851.5 \pm 223.8$ & $2613 \pm 99.5$ & $2454.1 \pm 84.7$ & $2413.6 \pm 157.5$ & $2462.2 \pm 63.3$ & $2505 \pm 156.4$ \\
\hline Caffeine & BfG & & $01012.8 \pm 287.6$ & $151.9 \pm 40.7$ & $128.8 \pm 17.8$ & $249.5 \pm 203.7$ & $101.5 \pm 122.1$ & $124.9 \pm 105.6$ & $84.1 \pm 22.7$ & $61.8 \pm 34.8$ \\
\hline Candesartan & Eawag & 350 & $02652.7 \pm 329.6$ & $2607.8 \pm 104.3$ & $2699.4 \pm 372.4$ & $2745.8 \pm 408.7$ & $2654.4 \pm 210.6$ & $2791.9 \pm 279.2$ & $2715.6 \pm 285.2$ & $2743.7 \pm 233.6$ \\
\hline Capecitabin & Eawag & & $02870.3 \pm 495.8$ & $621.7 \pm 212.8$ & $186.1 \pm 49.8$ & $152.3 \pm 45.7$ & $<\mathrm{LOQ}$ & $<\mathrm{LOQ}$ & $<\mathrm{LOQ}$ & $<\mathrm{LOQ}$ \\
\hline Carbanilide & BfG & & $5<$ LOQ & $<$ LOQ & $<\mathrm{LOQ}$ & $<\mathrm{LOQ}$ & $<\mathrm{LOQ}$ & $<\mathrm{LOQ}$ & $<\mathrm{LOQ}$ & $<\mathrm{LOQ}$ \\
\hline Carbendazim & $\mathrm{BfG}$ & & $57.4 \pm 0.7$ & $6.5 \pm 1$ & $6 \pm 0.5$ & $6.3 \pm 0.9$ & $5.4 \pm 0.3$ & $5.5 \pm 0$ & $6.1 \pm 0.4$ & $6.8 \pm 0.4$ \\
\hline Carbetamid & Eawag & & $02745.7 \pm 404.6$ & $2321.9 \pm 233.8$ & $2504.8 \pm 153.2$ & $2418.8 \pm 158.9$ & $1864.3 \pm 203.1$ & $1882.7 \pm 214.3$ & $1964.8 \pm 318.8$ & $1825.3 \pm 280$ \\
\hline CBZ & BfG & & $03306.7 \pm 749.3$ & $3022.2 \pm 391$ & $3320.4 \pm 473.8$ & $3414.5 \pm 136.4$ & $3000.5 \pm 393$ & $3281.8 \pm 393.1$ & $3531.9 \pm 249.3$ & $3037.2 \pm 709.2$ \\
\hline CBZ-10-OH & $\mathrm{BfG}$ & & $0801.1 \pm 130.5$ & $36.4 \pm 1.7$ & $<$ LOQ & $<$ LOQ & $<\mathrm{LOQ}$ & $<$ LOQ & $<$ LOQ & $<$ LOQ \\
\hline CBZ-2-OH & $\mathrm{BfG}$ & & $30646.9 \pm 134.6$ & $553.4 \pm 44$ & $554.7 \pm 35.3$ & $370.6 \pm 224.4$ & $72.5 \pm 31.1$ & $78.3 \pm 8.7$ & $64.4 \pm 0$ & $39.6 \pm 0$ \\
\hline $\mathrm{CBZ}-3-\mathrm{OH}$ & $\mathrm{BfG}$ & & $0723.2 \pm 157.2$ & $630.7 \pm 74.4$ & $641.2 \pm 49.9$ & $427.2 \pm 258.8$ & $77.7 \pm 35.5$ & $68 \pm 32.6$ & $53.8 \pm 20$ & $31.1 \pm 9.9$ \\
\hline Cetirizine & BfG & & $5309.4 \pm 110.1$ & $225.7 \pm 85.9$ & $220.8 \pm 58.3$ & $176.6 \pm 101.1$ & $25.4 \pm 7$ & $18 \pm 5$ & $14.4 \pm 3.6$ & $9.6 \pm 3.8$ \\
\hline Chlortoluron & Eawag & & $02616.2 \pm 453.7$ & $2405.3 \pm 147$ & $2541.6 \pm 160.1$ & $2363.8 \pm 178.7$ & $2154.4 \pm 191.2$ & $2064.3 \pm 31$ & $2101.7 \pm 179.2$ & $2065.5 \pm 157.7$ \\
\hline Citalopram & Eawag & 350 & $02989.4 \pm 410.9$ & $<\mathrm{LOQ}$ & $<\mathrm{LOQ}$ & $<\mathrm{LOQ}$ & $<\mathrm{LOQ}$ & $<\mathrm{LOQ}$ & $<\mathrm{LOQ}$ & $<\mathrm{LOQ}$ \\
\hline Clarithromycin & BfG & & $0100 \pm 74.2$ & $<\mathrm{LOQ}$ & $<L O Q$ & $<\mathrm{LOQ}$ & $<\mathrm{LOQ}$ & $<\mathrm{LOQ}$ & $<L O Q$ & $<L O Q$ \\
\hline Climbazole & $\mathrm{BfG}$ & & $26.2 \pm 4.2$ & $3.2 \pm 0$ & $7.2 \pm 0$ & $<L O Q$ & $<L O Q$ & $<L O Q$ & $<$ LOQ & $<$ LOQ \\
\hline Clofibrin-Acid & Eawag & & $01234.1 \pm 156.5$ & $757.1 \pm 70.9$ & $652.8 \pm 106.3$ & $492 \pm 72.1$ & $<\mathrm{LOQ}$ & $<\mathrm{LOQ}$ & $<\mathrm{LOQ}$ & $<L O Q$ \\
\hline Clopidogrel-Acid & BfG & & $0675.3 \pm 124.7$ & $488.2 \pm 20.5$ & $496.5 \pm 22$ & $315.8 \pm 172.5$ & $44.5 \pm 25.9$ & $37.3 \pm 16.4$ & $34.6 \pm 12$ & $18.9 \pm 8.6$ \\
\hline DEET & $\mathrm{BfG}$ & & $21098.1 \pm 335.8$ & $10.2 \pm 3.1$ & $3 \pm 0.2$ & $3.4 \pm 0.2$ & $2.3 \pm 0$ & $2.4 \pm 0.3$ & $3 \pm 0$ & $2.9 \pm 0.2$ \\
\hline Deprenyl & Eawag & & $02200.8 \pm 455.6$ & $707 \pm 266.5$ & $470.7 \pm 82.8$ & $231.5 \pm 12.7$ & $<\mathrm{LOQ}$ & $<\mathrm{LOQ}$ & $<\mathrm{LOQ}$ & $<\mathrm{LOQ}$ \\
\hline Diatrizoate & $\mathrm{BfG}$ & & $5470.6 \pm 177.7$ & $488.7 \pm 153.7$ & $383.6 \pm 164.7$ & $510.8 \pm 48.5$ & $461.6 \pm 139.5$ & $510.9 \pm 110.5$ & $432.6 \pm 122.8$ & $358.1 \pm 126.2$ \\
\hline Diclofenac & $\mathrm{BfG}$ & & $01908.6 \pm 429.3$ & $1153.8 \pm 341.1$ & $972.1 \pm 355.6$ & $601.8 \pm 430.7$ & $46.6 \pm 27.8$ & $44.3 \pm 18.6$ & $45.6 \pm 14$ & $29.2 \pm 12.9$ \\
\hline Diclofenac-4-OH & $\mathrm{BfG}$ & & $172.6 \pm 51.5$ & $39.7 \pm 31$ & $23.7 \pm 15.4$ & $16.9 \pm 12.6$ & $7.4 \pm 4.1$ & $5 \pm 2.4$ & $4.3 \pm 1.5$ & $2.1 \pm 0.2$ \\
\hline Diclofenac- $\mathrm{COOH}$ & $\mathrm{BfG}$ & & $543 \pm 22$ & $94.6 \pm 6$ & $107.5 \pm 18.6$ & $96.9 \pm 36.9$ & $95.6 \pm 49.7$ & $195.8 \pm 127.2$ & $171.7 \pm 4.2$ & $151.7 \pm 36.1$ \\
\hline Dimefuron & Eawag & 100 & $02360.8 \pm 405.9$ & $2137 \pm 88.4$ & $2273.8 \pm 260.1$ & $1399.8 \pm 387.3$ & $1189 \pm 131.1$ & $1178.1 \pm 66.6$ & $1200.8 \pm 67.4$ & $1251.4 \pm 75.7$ \\
\hline Dimethenamid & Eawag & & $02314.6 \pm 392.7$ & $2086.1 \pm 46.7$ & $2102.7 \pm 246.1$ & $1625.8 \pm 168$ & $1283.9 \pm 49.9$ & $1212.8 \pm 44.6$ & $1240.2 \pm 43.5$ & $1155.3 \pm 43.8$ \\
\hline Diuron & BfG & & $21240.8 \pm 411.5$ & $1153.6 \pm 323$ & $1189.2 \pm 268.2$ & $1203.4 \pm 175.6$ & $875.1 \pm 206.3$ & $1070.2 \pm 289.5$ & $1145.2 \pm 218.6$ & $876.2 \pm 313.1$ \\
\hline Emtricitabine & $\mathrm{BfG}$ & & $0258.1 \pm 48.8$ & $<$ LOQ & $<\mathrm{LOQ}$ & $<$ LOQ & $<$ LOQ & $<$ LOQ & $<$ LOQ & $<$ LOQ \\
\hline Enalaprilat & $\mathrm{BfG}$ & & $0<$ LOQ & $<L O Q$ & $<\mathrm{LOQ}$ & $<\mathrm{LOQ}$ & $<\mathrm{LOQ}$ & $<\mathrm{LOQ}$ & $<\mathrm{LOQ}$ & $<$ LOQ \\
\hline Ethofumesat & Eawag & 100 & $0357 \pm 49.4$ & $362.1 \pm 39.8$ & $320.1 \pm 31$ & $165.3 \pm 32.3$ & $106.7 \pm 4.4$ & $116 \pm 5.9$ & $116 \pm 0$ & $<$ LOQ \\
\hline Fenhexamid & Eawag & & $0129 \pm 41.2$ & $144.3 \pm 61$ & $130.3 \pm 45.2$ & $104.5 \pm 35.4$ & $70 \pm 16$ & $69 \pm 9$ & $76.5 \pm 4.5$ & $<$ LOQ \\
\hline Fexofenadine & BfG & & $5430.8 \pm 244.1$ & $15 \pm 9.9$ & $11.7 \pm 1.9$ & $<$ LOQ & $<$ LOQ & $<L O Q$ & $<$ LOQ & $<$ LOQ \\
\hline Flecainide & $\mathrm{BfG}$ & & $5308.5 \pm 134.9$ & $227.1 \pm 149.9$ & $193.3 \pm 86.2$ & $78.9 \pm 18.2$ & $<\mathrm{LOQ}$ & $<\mathrm{LOQ}$ & $<\mathrm{LOQ}$ & $<L O Q$ \\
\hline Fluconazole & $\mathrm{BfG}$ & & $2617.4 \pm 125.6$ & $564.1 \pm 73.6$ & $600.6 \pm 55$ & $607.3 \pm 32.3$ & $565.5 \pm 26.4$ & $576.3 \pm 23$ & $611.6 \pm 11.5$ & $561.3 \pm 91.1$ \\
\hline Flufenacet & Eawag & 50 & $0718.4 \pm 148.7$ & $661.8 \pm 83.5$ & $633.8 \pm 33$ & $226.3 \pm 54.5$ & $101.5 \pm 10$ & $81.3 \pm 2.9$ & $69.1 \pm 12.2$ & $61.3 \pm 0$ \\
\hline Furosemide & BfG & 20 & $02485.7 \pm 547.9$ & $1609.6 \pm 276.7$ & $1449.4 \pm 238$ & $999.3 \pm 713.9$ & $141.1 \pm 85.3$ & $84.1 \pm 19.8$ & $181.7 \pm 74.7$ & $172.5 \pm 84.8$ \\
\hline Gabapentin & $\mathrm{BfG}$ & & $51489.1 \pm 226.1$ & $202.9 \pm 76.3$ & $182.1 \pm 78.3$ & $164.9 \pm 111.5$ & $72.1 \pm 27.5$ & $37.9 \pm 13.2$ & $36.4 \pm 23$ & $20 \pm 14.4$ \\
\hline Hydrochlorothiazide & $\mathrm{BfG}$ & & $22770 \pm 761.4$ & $2778.1 \pm 664.4$ & $2330.8 \pm 576.3$ & $2556.4 \pm 218.4$ & $2280.8 \pm 299.5$ & $2206.8 \pm 214.5$ & $2207.9 \pm 319.3$ & $2041.8 \pm 402$ \\
\hline Ibuprofen & $\mathrm{BfG}$ & 50 & $0250.6 \pm 121.6$ & $<\mathrm{LOQ}$ & $<\mathrm{LOQ}$ & $<\mathrm{LOQ}$ & $<\mathrm{LOQ}$ & $<\mathrm{LOQ}$ & $<\mathrm{LOQ}$ & $<\mathrm{LOQ}$ \\
\hline lopamidol & $\mathrm{BfG}$ & & $544.8 \pm 23.7$ & $45.8 \pm 19.5$ & $25.3 \pm 21$ & $32 \pm 14.6$ & $36.5 \pm 16.2$ & $29.4 \pm 17.2$ & $35.9 \pm 1.6$ & $25.5 \pm 10$ \\
\hline lopromide & $\mathrm{BfG}$ & 10 & $0101.4 \pm 44.8$ & $14.6 \pm 0$ & $<$ LOQ & $<$ LOQ & $<$ LOQ & $<\mathrm{LOQ}$ & $<$ LOQ & $<\mathrm{LOQ}$ \\
\hline Iprovalicarb & Eawag & 50 & $02297 \pm 388.5$ & $193.3 \pm 81.1$ & $140.2 \pm 48.4$ & $82.5 \pm 4.5$ & $<\mathrm{LOQ}$ & $<L O Q$ & $<\mathrm{LOQ}$ & $<$ LOQ \\
\hline Irgarol & Eawag & 100 & $0<\mathrm{LOQ}$ & $<\mathrm{LOQ}$ & $<$ LOQ & $<\mathrm{LOQ}$ & $<\mathrm{LOQ}$ & $<\mathrm{LOQ}$ & $<\mathrm{LOQ}$ & $<\mathrm{LOQ}$ \\
\hline Isoproturon & Eawag & 50 & $02907.6 \pm 457.7$ & $2685.3 \pm 100.5$ & $2746 \pm 176.3$ & $2433.3 \pm 146.8$ & $2164.6 \pm 151.1$ & $2102.4 \pm 23.1$ & $2168 \pm 116$ & $2117.1 \pm 154.5$ \\
\hline Ketoprofen & Eawag & 50 & $0 \quad 1528.2 \pm 182.5$ & $139.3 \pm 23.7$ & $63.5 \pm 1.5$ & $<$ LOQ & $<$ LOQ & $<$ LOQ & $<$ LOQ & $<\mathrm{LOQ}$ \\
\hline Lamotrigine & BfG & 20 & $0763.3 \pm 214.8$ & $850.5 \pm 277.5$ & $869.8 \pm 195.8$ & $971.5 \pm 98.1$ & $800.4 \pm 132.8$ & $803 \pm 174.4$ & $1112.2 \pm 183.9$ & $1110.4 \pm 156.9$ \\
\hline Levetiracetam & $\mathrm{BfG}$ & & $02261.5 \pm 330.9$ & $<$ LOQ & $<$ LOQ & $<L O Q$ & $<$ LOQ & $<L O Q$ & $<$ LOQ & $<\mathrm{LOQ}$ \\
\hline Lidocaine & Eawag & 50 & $03872 \pm 642.4$ & $3456.5 \pm 164.5$ & $3580.5 \pm 301.9$ & $2843.3 \pm 315.5$ & $2160.3 \pm 122$ & $2093.9 \pm 228.8$ & $2135.8 \pm 178.7$ & $2218.2 \pm 176.8$ \\
\hline Mecoprop & $B f G$ & 10 & $0748.6 \pm 116.3$ & $231.8 \pm 47$ & $130.9 \pm 41.2$ & $111.7 \pm 53.7$ & $<\mathrm{LOQ}$ & $<\mathrm{LOQ}$ & $<\mathrm{LOQ}$ & $<\mathrm{LOQ}$ \\
\hline Metolachlor & Eawag & 100 & $0915.8 \pm 140.1$ & $891.9 \pm 44.1$ & $889 \pm 42.7$ & $454.8 \pm 99.3$ & $375.1 \pm 48.2$ & $354.3 \pm 14.9$ & $345.6 \pm 18$ & $314.6 \pm 20.3$ \\
\hline Metoprolol & BfG & & $51297.6 \pm 236.6$ & $74.4 \pm 29.6$ & $50.1 \pm 23.2$ & $47.7 \pm 18$ & $31.9 \pm 5.6$ & $<L O Q$ & $<$ LOQ & $<L O Q$ \\
\hline Metoxuron & Eawag & 50 & $03022.5 \pm 414.2$ & $2726.3 \pm 251.1$ & $2681.8 \pm 166.4$ & $2833.5 \pm 190.9$ & $2596.3 \pm 226.3$ & $2634.7 \pm 14.8$ & $2558.9 \pm 209.7$ & $2530.2 \pm 254.5$ \\
\hline Mianserin & Eawag & 350 & $01193.4 \pm 290.1$ & $410 \pm 51.4$ & $359.5 \pm 9.5$ & $<\mathrm{LOQ}$ & $<\mathrm{LOQ}$ & $<\mathrm{LOQ}$ & $<\mathrm{LOQ}$ & $<\mathrm{LOQ}$ \\
\hline Monuron & Eawag & 50 & $02895 \pm 524$ & $2718.3 \pm 115.6$ & $2823.3 \pm 119.9$ & $2693 \pm 138.5$ & $2521.1 \pm 212.9$ & $2461.4 \pm 34.1$ & $2520.6 \pm 215.3$ & $2439.1 \pm 165.2$ \\
\hline Naproxen & Eawag & 200 & $0767.9 \pm 187.6$ & $<$ LOQ & $<$ LOQ & $293 \pm 0$ & $<L O Q$ & $<$ LOQ & $<$ LOQ & $<\mathrm{LOQ}$ \\
\hline Olmesartan & Eawag & 50 & $03572.9 \pm 556.5$ & $3473.9 \pm 164.5$ & $3209.3 \pm 357.4$ & $3390.5 \pm 236.6$ & $3163.1 \pm 150.4$ & $3183.1 \pm 292.9$ & $3159.4 \pm 22.5$ & $3215.9 \pm 319.3$ \\
\hline Oxazepam & $\mathrm{BfG}$ & 20 & $034.6 \pm 9$ & $34.4 \pm 0$ & $23.1 \pm 0$ & $22.2 \pm 0$ & $<$ LOQ & $<L O Q$ & $<\mathrm{LOQ}$ & $<L O Q$ \\
\hline Oxypurinol & BfG & & $2901.5 \pm 278.2$ & $825.6 \pm 145.3$ & $850.6 \pm 161$ & $921.6 \pm 39.9$ & $944.2 \pm 118.5$ & $1095.2 \pm 125.2$ & $1593.9 \pm 512.1$ & $1313.7 \pm 271.1$ \\
\hline Paracetamol & Eawag & 350 & $01569.1 \pm 219$ & $<\mathrm{LOQ}$ & $<\mathrm{LOQ}$ & $<\mathrm{LOQ}$ & $<\mathrm{LOQ}$ & $<\mathrm{LOQ}$ & $<\mathrm{LOQ}$ & $<\mathrm{LOQ}$ \\
\hline Pargyline & Eawag & 50 & $01293.2 \pm 211.8$ & $359.5 \pm 124.6$ & $295.3 \pm 107.2$ & $140.3 \pm 33.5$ & $<L O Q$ & $<\mathrm{LOQ}$ & $<$ LOQ & $<L O Q$ \\
\hline Pheniramine & Eawag & 500 & $04265.1 \pm 753.3$ & $1168.3 \pm 125.5$ & $1476.9 \pm 93.2$ & $1449.8 \pm 60$ & $<L O Q$ & $<L O Q$ & $<L O Q$ & $583 \pm 0$ \\
\hline Pregabalin & BfG & 20 & $0884.6 \pm 157.1$ & $57.2 \pm 18.4$ & $36.3 \pm 2.5$ & $40.1 \pm 1.9$ & $26.5 \pm 0$ & $39.9 \pm 0$ & $<\mathrm{LOQ}$ & $<\mathrm{LOQ}$ \\
\hline Primidone & BfG & 20 & $095.4 \pm 26.1$ & $97.1 \pm 21.7$ & $88 \pm 22.1$ & $98.4 \pm 6.6$ & $90.5 \pm 16.5$ & $90.5 \pm 11.7$ & $91.8 \pm 14.1$ & $82.2 \pm 17$ \\
\hline Propachlor & Eawag & 50 & $01593.7 \pm 183.4$ & $121.8 \pm 43.2$ & $77.3 \pm 10.3$ & $<L O Q$ & $<L O Q$ & $<L O Q$ & $<L O Q$ & $<L O Q$ \\
\hline Ramipril & $\mathrm{BfG}$ & 10 & $012.4 \pm 0.9$ & $<L O Q$ & $<L O Q$ & $<$ LOQ & $<L O Q$ & $<$ LOQ & $<$ LOQ & $<$ LOQ \\
\hline Ramiprilat & $\mathrm{BfG}$ & 20 & $0606.3 \pm 101.2$ & $57.3 \pm 29.2$ & $35.1 \pm 2.3$ & $44.5 \pm 0$ & $<L O Q$ & $<L O Q$ & $<$ LOQ & $<$ LOQ \\
\hline Ranitidin & Eawag & 200 & $03550.2 \pm 616.4$ & $1040 \pm 100.4$ & $427.5 \pm 22.7$ & $395 \pm 17$ & $<\mathrm{LOQ}$ & $<\mathrm{LOQ}$ & $<\mathrm{LOQ}$ & $<\mathrm{LOQ}$ \\
\hline Rufinamid & Eawag & 50 & $01473.3 \pm 209.4$ & $<\mathrm{LOQ}$ & $<\mathrm{LOQ}$ & $<\mathrm{LOQ}$ & $<\mathrm{LOQ}$ & $<\mathrm{LOQ}$ & $<\mathrm{LOQ}$ & $<\mathrm{LOQ}$ \\
\hline Saccharin & Eawag & 100 & $0753.2 \pm 199.3$ & $131.5 \pm 4.8$ & $134.9 \pm 5.5$ & $136 \pm 8.6$ & $181.5 \pm 61$ & $130.3 \pm 5.3$ & $138.3 \pm 13$ & $157.2 \pm 34.7$ \\
\hline Saluamine & BfG & 100 & $0118.7 \pm 14$ & $325.2 \pm 79$ & $447.4 \pm 149.6$ & $337.4 \pm 233.5$ & $232.6 \pm 159$ & $338.6 \pm 15.2$ & $520.1 \pm 61.6$ & $432.6 \pm 50.9$ \\
\hline Sitagliptin & $\mathrm{BfG}$ & & $5749.4 \pm 307$ & $484.5 \pm 257.3$ & $500.1 \pm 233$ & $632.6 \pm 167.2$ & $<\mathrm{LOQ}$ & $<\mathrm{LOQ}$ & $<\mathrm{LOQ}$ & $<\mathrm{LOQ}$ \\
\hline Sotalol & Eawag & 350 & $03732.5 \pm 891.6$ & $1746.9 \pm 550.5$ & $1398.5 \pm 431.4$ & $1365.5 \pm 325.2$ & $500.7 \pm 84.5$ & $397 \pm 0$ & $<L O Q$ & $<L O Q$ \\
\hline Sucralose & BfG & 20 & $02908 \pm 1530.9$ & $2809.3 \pm 1043.3$ & $2390.2 \pm 281.6$ & $2659.3 \pm 1082.7$ & $2678.6 \pm 894.1$ & $3438.8 \pm 1239.2$ & $3715.8 \pm 822.7$ & $4029.7 \pm 1701.1$ \\
\hline Sulfadiazin & Eawag & 50 & $03361.9 \pm 657.2$ & $341.1 \pm 198.7$ & $289.2 \pm 174.1$ & $289.3 \pm 145.8$ & $<$ LOQ & $<$ LOQ & $61 \pm 0$ & $<\mathrm{LOQ}$ \\
\hline Sulfamethazin & Eawag & & $03282.1 \pm 564.6$ & $2578.3 \pm 126.4$ & $2597.7 \pm 176.7$ & $2432.5 \pm 115.5$ & $1963.9 \pm 147.1$ & $1832 \pm 13.1$ & $1877.2 \pm 205.6$ & $1802.7 \pm 151.9$ \\
\hline
\end{tabular}


$11.3 \pm 0$

$3283.6 \pm 108$

$1882 \pm 265.5 \quad 851.1 \pm 23.6$

$546 \pm 24.3$

$493.2 \pm 60.9$

BfG
Eawag

Valsartan

$2910.3 \pm 95.7 \quad 6 \pm 2$

$54.3 \pm 0$

$135.3 \pm 0$

$94 \pm 13.7$

$851.1 \pm 97$

$166.5 \pm 11.9 \quad 135.4 \pm 34.5$

Venlafaxine

$203436.6 \pm 723.4 \quad 1760.6 \pm 334$

$3.3 \pm 0.8$

$<$ LOQ

$5544 \pm 112.2 \quad 495.8 \pm 19.1$

$3.5 \pm 0<<\mathrm{LOQ}$

$<$ LOQ

$<$ LOQ

$<$ LOQ

$<$ LOQ

$<$ LOQ 


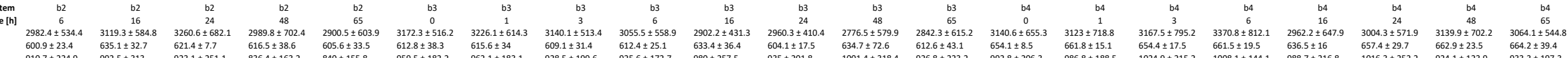

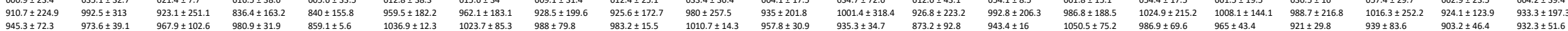

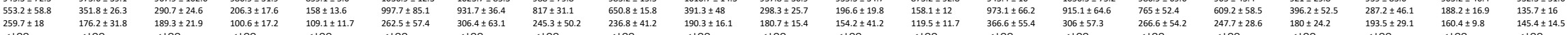

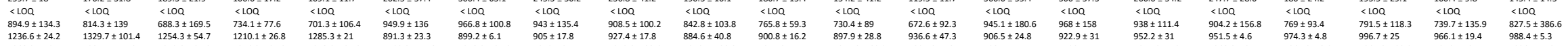

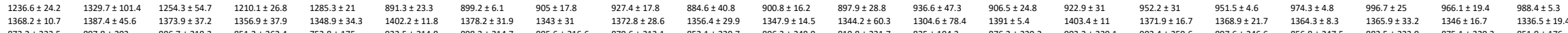

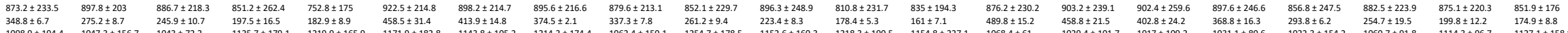

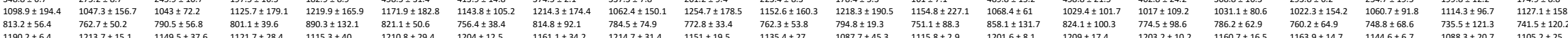

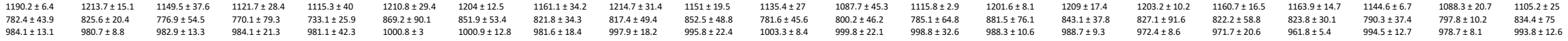

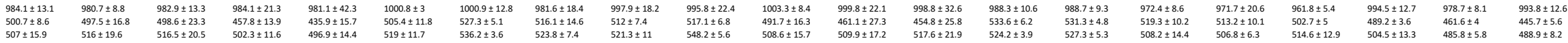

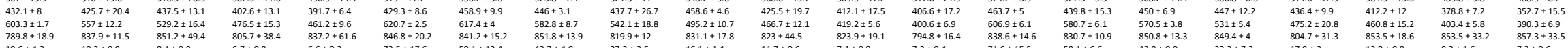

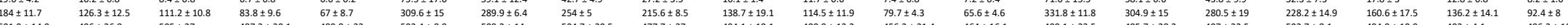

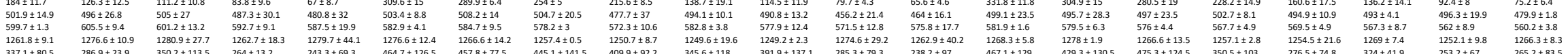

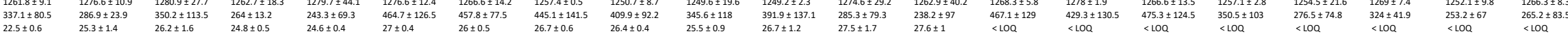

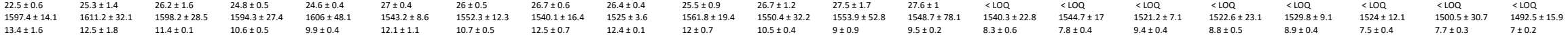

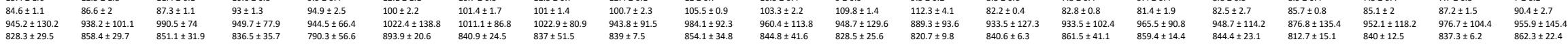

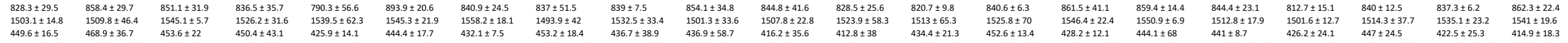

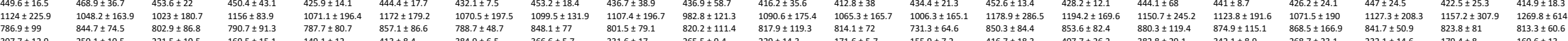

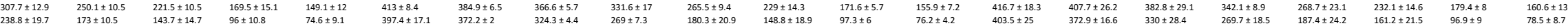

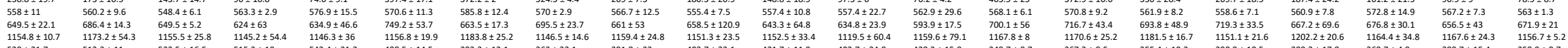

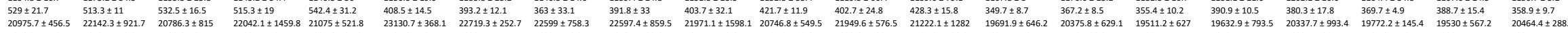

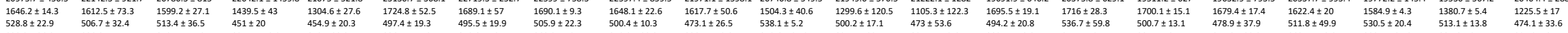

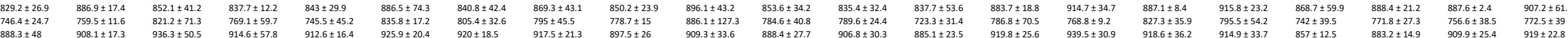

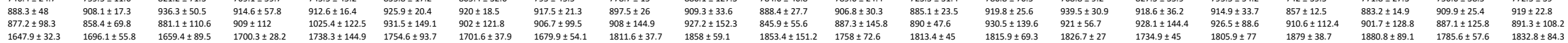

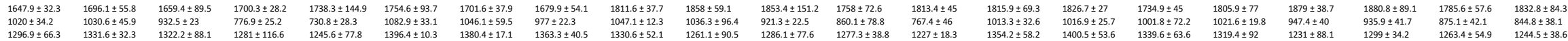

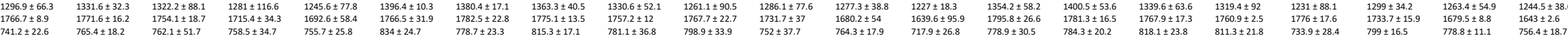

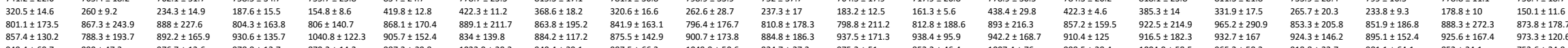

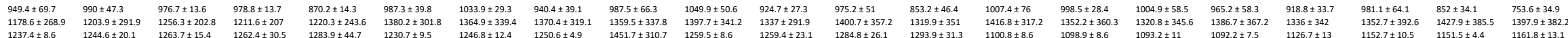

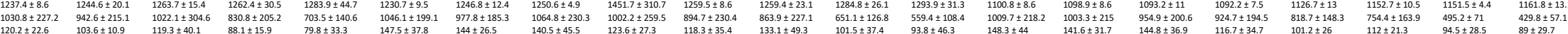

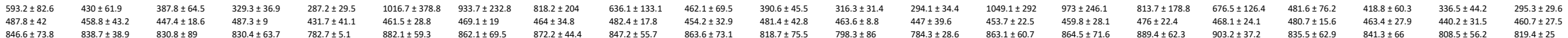

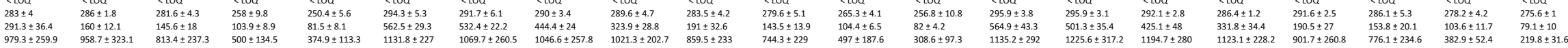

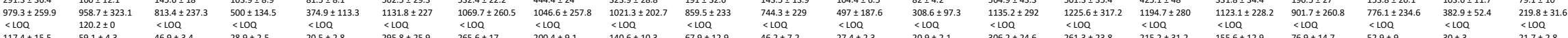

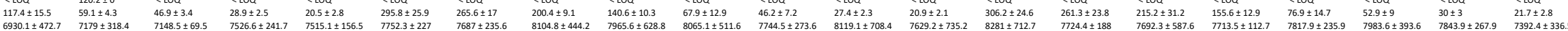

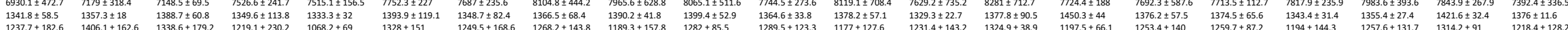

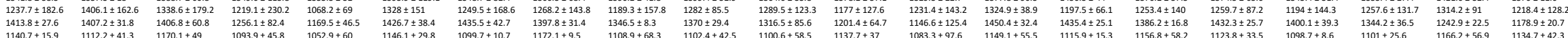

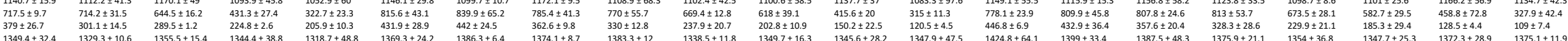

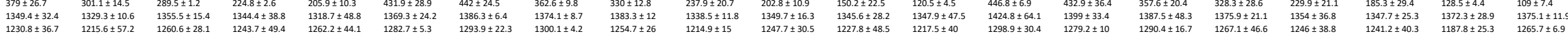

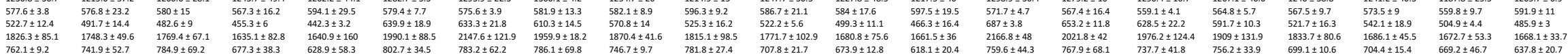

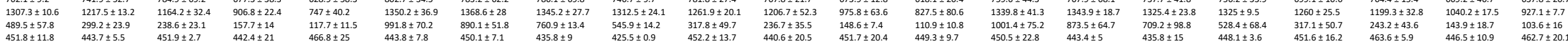




\section{S13: Measured concentrations [ng/L] of trace organic chemicals in batch sorption test}

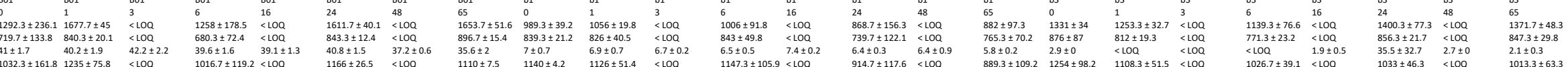

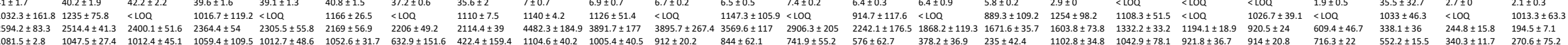

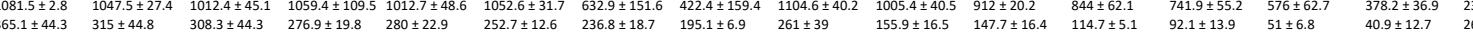

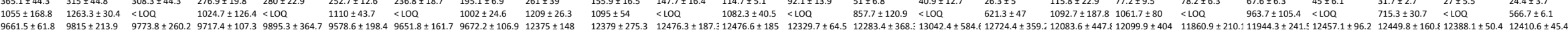

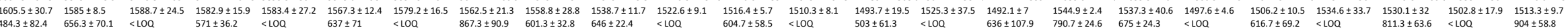

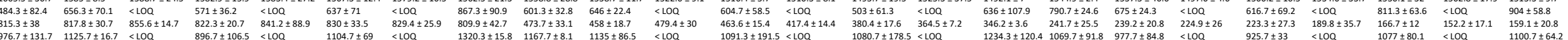

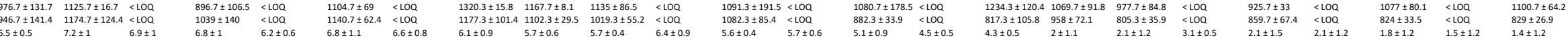

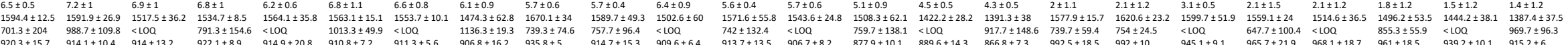

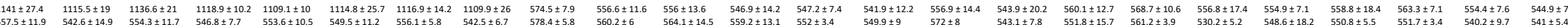

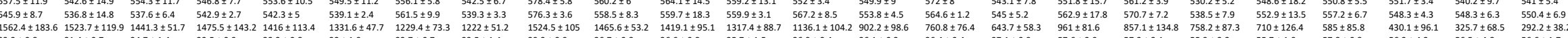

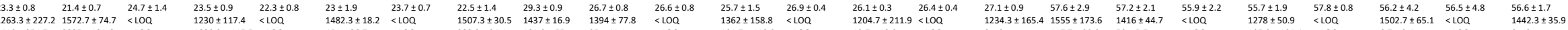

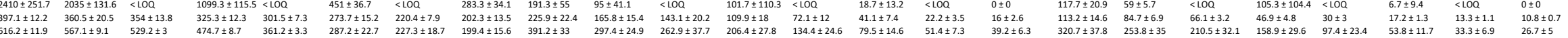

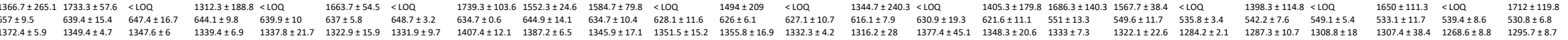

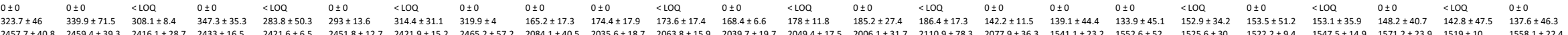

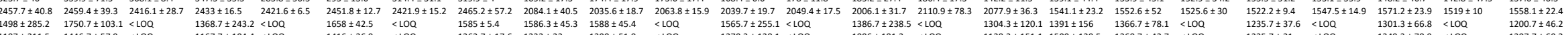

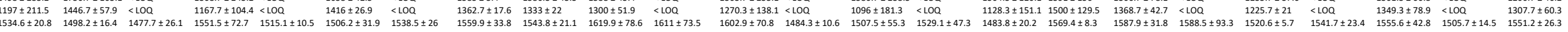

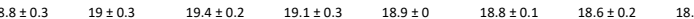

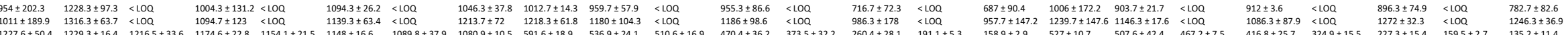

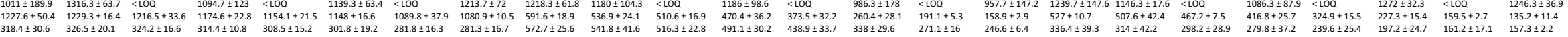

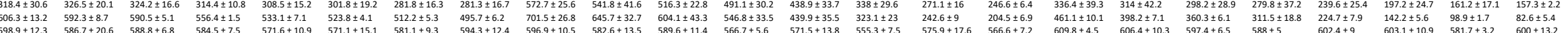

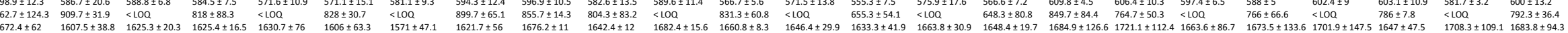

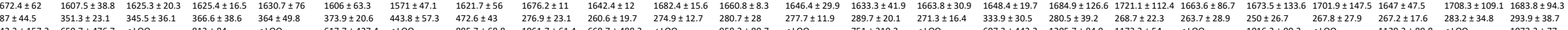

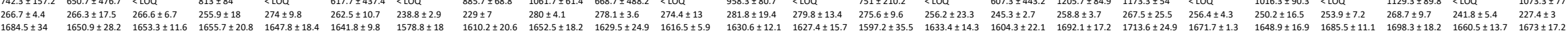

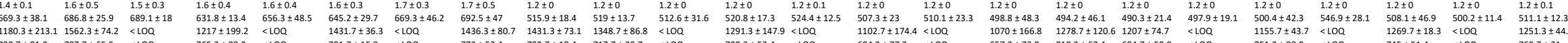

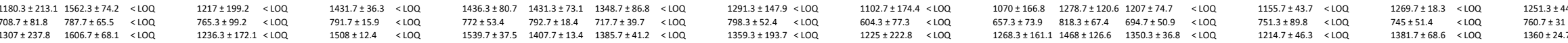

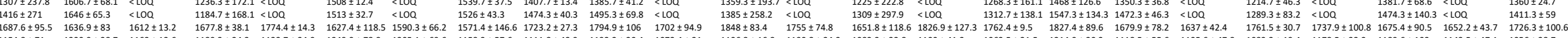

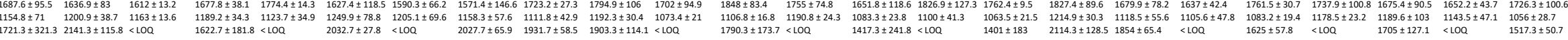

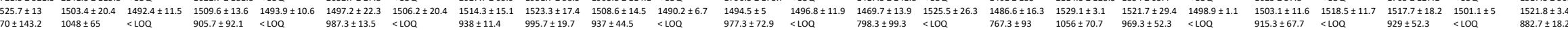

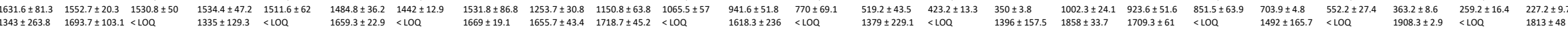

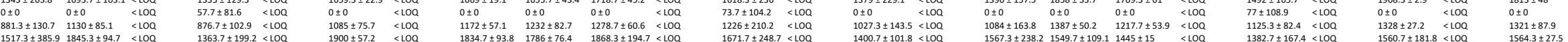

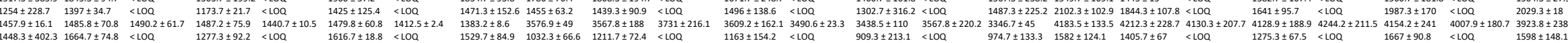

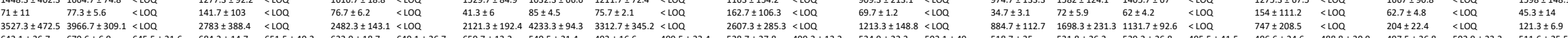

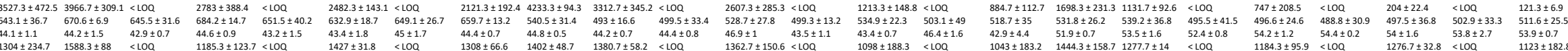

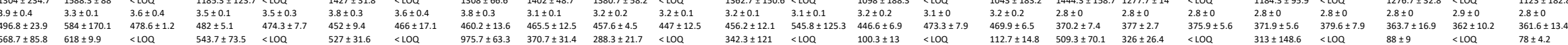

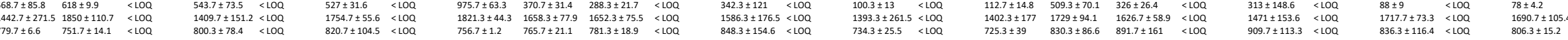

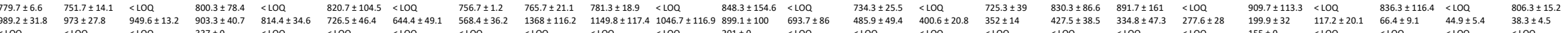

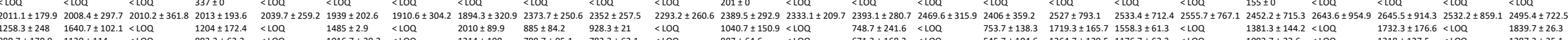

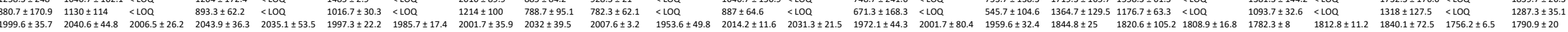

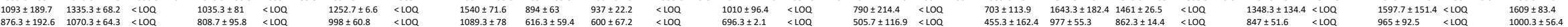

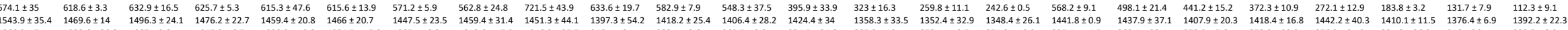

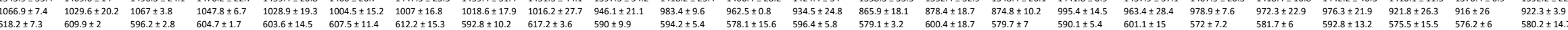


S14 Determined rate constants from batch biotransformation experiments

\begin{tabular}{|c|c|c|c|c|c|c|c|c|c|c|c|c|c|c|c|c|}
\hline \multirow[b]{2}{*}{ Compound } & \multirow[b]{2}{*}{ Laboratory } & \multicolumn{5}{|c|}{$\operatorname{kbiol}\left(\mathrm{d}^{-1}\right)$} & \multicolumn{5}{|c|}{ Standard error (SE) } & \multicolumn{5}{|c|}{ Standard deviation (SD) } \\
\hline & & B01 & b1 & b2 & b3 & b4 & B01 & b1 & b2 & b3 & b4 & B01 & b1 & b2 & b3 & b4 \\
\hline Acesulfame & Eawag & 1,113 & $-0,020$ & $-0,007$ & $-0,004$ & $-0,004$ & 0,050 & 0,050 & 0,050 & 0,050 & 0,050 & 0,236 & 0,236 & 0,236 & 0,236 & 0,236 \\
\hline Acetamiprid & Eawag & 0,195 & 0,271 & 0,019 & 0,004 & 0,031 & 0,024 & 0,024 & 0,024 & 0,024 & 0,024 & 0,120 & 0,120 & 0,120 & 0,120 & 0,120 \\
\hline Alachlor & Eawag & 0,254 & 0,069 & 0,028 & 0,053 & 0,029 & 0,023 & 0,023 & 0,023 & 0,023 & 0,023 & 0,114 & 0,114 & 0,114 & 0,114 & 0,114 \\
\hline Benzotriazole & BfG & $-0,007$ & 0,315 & $-0,008$ & $-0,008$ & $-0,022$ & 0,006 & 0,006 & 0,006 & 0,006 & 0,006 & 0,029 & 0,029 & 0,029 & 0,029 & 0,029 \\
\hline Bezafibrate & $\mathrm{BfG}$ & 0,994 & 0,212 & 0,010 & 0,018 & 0,014 & 0,097 & 0,097 & 0,097 & 0,097 & 0,097 & 0,475 & 0,475 & 0,475 & 0,475 & 0,475 \\
\hline Caffeine & BfG & 1,295 & 0,498 & 0,350 & 0,370 & 0,365 & 0,039 & 0,039 & 0,039 & 0,039 & 0,039 & 0,165 & 0,165 & 0,165 & 0,165 & 0,165 \\
\hline Capecitabin & Eawag & 0,764 & 0,187 & $-0,023$ & 0,015 & 0,043 & 0,049 & 0,049 & 0,049 & 0,049 & 0,049 & 0,240 & 0,240 & 0,240 & 0,240 & 0,240 \\
\hline CBZ-10-OH & BfG & 0,925 & 0,261 & 0,067 & 0,052 & 0,064 & 0,036 & 0,036 & 0,036 & 0,036 & 0,036 & 0,176 & 0,176 & 0,176 & 0,176 & 0,176 \\
\hline CBZ-2-OH & BfG & 0,068 & 0,006 & 0,021 & 0,011 & 0,026 & 0,007 & 0,007 & 0,007 & 0,007 & 0,007 & 0,034 & 0,034 & 0,034 & 0,034 & 0,034 \\
\hline CBZ-3-OH & $\mathrm{BfG}$ & 0,140 & 0,065 & 0,052 & 0,036 & 0,094 & 0,008 & 0,008 & 0,008 & 0,008 & 0,008 & 0,039 & 0,039 & 0,039 & 0,039 & 0,039 \\
\hline Clofibrin-Acid & Eawag & 0,295 & 0,305 & 0,010 & 0,034 & 0,010 & 0,019 & 0,019 & 0,019 & 0,019 & 0,019 & 0,094 & 0,094 & 0,094 & 0,094 & 0,094 \\
\hline Clopidogrel-Acid & BfG & 0,200 & 0,174 & 0,013 & 0,005 & 0,012 & 0,014 & 0,014 & 0,014 & 0,014 & 0,014 & 0,069 & 0,069 & 0,069 & 0,069 & 0,069 \\
\hline DEET & $\mathrm{BfG}$ & 1,054 & 0,237 & 0,007 & $-0,001$ & 0,002 & 0,058 & 0,058 & 0,058 & 0,058 & 0,058 & 0,284 & 0,284 & 0,284 & 0,284 & 0,284 \\
\hline Diclofenac & BfG & 0,493 & 0,162 & 0,004 & $-0,002$ & 0,011 & 0,031 & 0,031 & 0,031 & 0,031 & 0,031 & 0,152 & 0,152 & 0,152 & 0,152 & 0,152 \\
\hline Dimefuron & Eawag & 0,033 & 0,013 & $-0,003$ & 0,042 & $-0,010$ & 0,012 & 0,012 & 0,012 & 0,012 & 0,012 & 0,059 & 0,059 & 0,059 & 0,059 & 0,059 \\
\hline Dimethenamid & Eawag & 0,068 & 0,003 & 0,010 & 0,016 & $-0,002$ & 0,013 & 0,013 & 0,013 & 0,013 & 0,013 & 0,062 & 0,062 & 0,062 & 0,062 & 0,062 \\
\hline Emtricitabine & BfG & 1,006 & 0,125 & 0,020 & 0,017 & 0,021 & 0,055 & 0,055 & 0,055 & 0,055 & 0,055 & 0,264 & 0,264 & 0,264 & 0,264 & 0,264 \\
\hline Fenhexamid & Eawag & 0,117 & 0,075 & 0,018 & 0,034 & 0,022 & 0,014 & 0,014 & 0,014 & 0,014 & 0,014 & 0,069 & 0,069 & 0,069 & 0,069 & 0,069 \\
\hline Flufenacet & Eawag & 0,067 & 0,091 & 0,033 & 0,055 & 0,024 & 0,023 & 0,023 & 0,023 & 0,023 & 0,023 & 0,112 & 0,112 & 0,112 & 0,112 & 0,112 \\
\hline Furosemide & BfG & 0,367 & 0,168 & 0,007 & 0,007 & 0,004 & 0,024 & 0,024 & 0,024 & 0,024 & 0,024 & 0,118 & 0,118 & 0,118 & 0,118 & 0,118 \\
\hline Gabapentin & $\mathrm{BfG}$ & 0,936 & 0,350 & 0,000 & $-0,031$ & $-0,006$ & 0,036 & 0,036 & 0,036 & 0,036 & 0,036 & 0,176 & 0,176 & 0,176 & 0,176 & 0,176 \\
\hline Ibuprofen & BfG & 5,235 & 2,776 & 0,095 & 0,158 & 0,120 & 0,275 & 0,275 & 0,275 & 0,275 & 0,275 & 1,065 & 1,065 & 1,065 & 1,065 & 1,065 \\
\hline Iopromide & $\mathrm{BfG}$ & 0,959 & 0,362 & 0,049 & 0,013 & 0,012 & 0,038 & 0,038 & 0,038 & 0,038 & 0,038 & 0,182 & 0,182 & 0,182 & 0,182 & 0,182 \\
\hline Iprovalicarb & Eawag & 1,170 & 0,095 & 0,018 & 0,014 & 0,000 & 0,066 & 0,066 & 0,066 & 0,066 & 0,066 & 0,305 & 0,305 & 0,305 & 0,305 & 0,305 \\
\hline Ketoprofen & Eawag & 1,091 & 0,893 & $-0,053$ & 0,012 & 0,018 & 0,071 & 0,071 & 0,071 & 0,071 & 0,071 & 0,332 & 0,332 & 0,332 & 0,332 & 0,332 \\
\hline Levetiracetam & BfG & 1,537 & 0,650 & 0,139 & 0,113 & 0,071 & 0,076 & 0,076 & 0,076 & 0,076 & 0,076 & 0,331 & 0,331 & 0,331 & 0,331 & 0,331 \\
\hline Mecoprop & BfG & 0,419 & 0,605 & 0,023 & 0,030 & 0,030 & 0,028 & 0,028 & 0,028 & 0,028 & 0,028 & 0,137 & 0,137 & 0,137 & 0,137 & 0,137 \\
\hline Metolachlor & Eawag & 0,021 & 0,005 & 0,008 & 0,039 & 0,015 & 0,015 & 0,015 & 0,015 & 0,015 & 0,015 & 0,073 & 0,073 & 0,073 & 0,073 & 0,073 \\
\hline Naproxen & Eawag & 1,102 & 0,535 & 0,043 & 0,044 & 0,096 & 0,074 & 0,074 & 0,074 & 0,074 & 0,074 & 0,316 & 0,316 & 0,316 & 0,316 & 0,316 \\
\hline Paracetamol & Eawag & 4,030 & 0,592 & 0,144 & 0,230 & 0,322 & 0,653 & 0,653 & 0,653 & 0,653 & 0,653 & 2,066 & 2,066 & 2,066 & 2,066 & 2,066 \\
\hline Pregabalin & BfG & 0,667 & 0,251 & 0,019 & 0,013 & 0,011 & 0,033 & 0,033 & 0,033 & 0,033 & 0,033 & 0,162 & 0,162 & 0,162 & 0,162 & 0,162 \\
\hline Ramiprilat & BfG & 1,388 & 0,883 & 0,057 & 0,049 & 0,025 & 0,058 & 0,058 & 0,058 & 0,058 & 0,058 & 0,259 & 0,259 & 0,259 & 0,259 & 0,259 \\
\hline Rufinamid & Eawag & 2,388 & 1,068 & 0,392 & 0,466 & 0,615 & 0,088 & 0,088 & 0,088 & 0,088 & 0,088 & 0,373 & 0,373 & 0,373 & 0,373 & 0,373 \\
\hline Sulfadiazin & Eawag & 0,278 & 0,005 & 0,015 & 0,008 & 0,000 & 0,026 & 0,026 & 0,026 & 0,026 & 0,026 & 0,127 & 0,127 & 0,127 & 0,127 & 0,127 \\
\hline Sulfamethoxazol & BfG & 0,800 & 0,046 & 0,077 & 0,081 & 0,073 & 0,044 & 0,044 & 0,044 & 0,044 & 0,044 & 0,216 & 0,216 & 0,216 & 0,216 & 0,216 \\
\hline Sulfapyridin & Eawag & 0,261 & 0,005 & 0,026 & 0,012 & $-0,004$ & 0,018 & 0,018 & 0,018 & 0,018 & 0,018 & 0,086 & 0,086 & 0,086 & 0,086 & 0,086 \\
\hline Sulfathiazol & Eawag & 1,532 & 0,321 & 0,317 & 0,354 & 0,330 & 0,078 & 0,078 & 0,078 & 0,078 & 0,078 & 0,333 & 0,333 & 0,333 & 0,333 & 0,333 \\
\hline Terbuthylazine & BfG & 0,030 & 0,000 & 0,017 & 0,009 & 0,007 & 0,012 & 0,012 & 0,012 & 0,012 & 0,012 & 0,059 & 0,059 & 0,059 & 0,059 & 0,059 \\
\hline Terbutryn & BfG & 0,088 & 0,064 & 0,008 & 0,021 & 0,017 & 0,009 & 0,009 & 0,009 & 0,009 & 0,009 & 0,044 & 0,044 & 0,044 & 0,044 & 0,044 \\
\hline Trinexapac-ethyl & Eawag & 0,654 & 0,242 & 0,074 & 0,087 & 0,063 & 0,024 & 0,024 & 0,024 & 0,024 & 0,024 & 0,119 & 0,119 & 0,119 & 0,119 & 0,119 \\
\hline Valsartan & BfG & 2,056 & 0,798 & 0,221 & 0,182 & 0,137 & 0,087 & 0,087 & 0,087 & 0,087 & 0,087 & 0,417 & 0,417 & 0,417 & 0,417 & 0,417 \\
\hline
\end{tabular}


SI5: Gene copy numbers in the sands originating from the different columns

\begin{tabular}{|c|c|c|c|c|c|c|c|}
\hline column & replicate & $\begin{array}{l}\text { weighing } \\
\text { [g] }\end{array}$ & $\begin{array}{l}\text { elution volume } \\
\qquad[\mu \mathrm{L}]\end{array}$ & $\begin{array}{l}\text { DNA concentration } \\
{[\mathrm{ng} / \mu \mathrm{L}]}\end{array}$ & gene copies $/ \mu \mathrm{L}$ & SD & $\begin{array}{c}\text { gene } \\
\text { copies/g }\end{array}$ \\
\hline B01 & 1 & 1 & 65 & 140 & $3,92 E+07$ & $4,47 E+06$ & $2,55 E+09$ \\
\hline B01 & 2 & 1 & 65 & 128 & $3,44 \mathrm{E}+07$ & $1,10 \mathrm{E}+07$ & $2,23 E+09$ \\
\hline B01 & 3 & 1 & 65 & 171 & $6,11 \mathrm{E}+07$ & $9,48 \mathrm{E}+06$ & $3,97 E+09$ \\
\hline b1 & 1 & 1 & 65 & 39,5 & $5,75 E+06$ & $1,82 E+05$ & $3,74 \mathrm{E}+08$ \\
\hline b1 & 2 & 1 & 65 & 39 & $4,02 E+06$ & $2,60 E+06$ & $2,61 E+08$ \\
\hline b1 & 3 & 1 & 65 & 66 & 7,97E+06 & $8,77 E+05$ & $5,18 \mathrm{E}+08$ \\
\hline b2 & 1 & 1 & 65 & 32,5 & $1,29 E+06$ & $9,35 E+04$ & $8,41 E+07$ \\
\hline b2 & 2 & 1 & 65 & 38 & $1,38 \mathrm{E}+06$ & $8,05 E+04$ & $8,99 E+07$ \\
\hline b2 & 3 & 1 & 65 & 47 & $1,18 \mathrm{E}+06$ & $2,10 E+04$ & $7,66 \mathrm{E}+07$ \\
\hline b3 & 1 & 1 & 65 & 49 & $1,06 E+06$ & $3,80 E+04$ & $6,90 E+07$ \\
\hline b3 & 2 & 1 & 65 & 32,5 & $1,05 E+06$ & $4,20 E+04$ & $6,80 \mathrm{E}+07$ \\
\hline b3 & 3 & 1 & 65 & 48,5 & $1,31 E+06$ & $1,94 \mathrm{E}+05$ & $8,49 E+07$ \\
\hline b4 & 1 & 1 & 65 & 28 & $7,53 E+05$ & $1,52 E+05$ & $4,89 E+07$ \\
\hline b4 & 2 & 1 & 65 & 31 & $7,52 \mathrm{E}+05$ & $1,40 E+04$ & $4,89 E+07$ \\
\hline b4 & 3 & 1 & 65 & 49,5 & $3,36 E+05$ & $6,95 E+04$ & $2,18 E+07$ \\
\hline
\end{tabular}


S16: Removal of TOrCs in column and batch experiments

\begin{tabular}{|c|c|c|c|c|c|c|c|}
\hline \multirow[b]{2}{*}{ Compound } & \multirow[b]{2}{*}{$\begin{array}{c}\text { charge at } \\
\text { pH7 }\end{array}$} & \multirow[b]{2}{*}{$\log \mathrm{D}$} & \multirow[b]{2}{*}{$\begin{array}{c}\text { WWTP effl. } \\
\mathrm{ng} / \mathrm{L}\end{array}$} & \multicolumn{2}{|c|}{ column experiments } & \multicolumn{2}{|c|}{ batch experiments } \\
\hline & & & & $\begin{array}{c}\mathrm{c}_{0} \\
\mathrm{ng} / \mathrm{L}\end{array}$ & $c / c_{\max }$ & $\begin{array}{l}\text { sorption [\%] } \\
\text { B01 b1 b3 }\end{array}$ & $\begin{array}{c}\text { bio [\%] } \\
\text { B01 b1 b3 }\end{array}$ \\
\hline \multicolumn{8}{|c|}{ Group 1: persistent substances (< $30 \%$ removal after $7.8 \mathrm{~d})$} \\
\hline 5-Methyl-Benzotriazol & neutral & 1,8 & 385 & 935 & 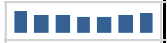 & n.a. & $-\ldots$ \\
\hline${ }^{2}$ Bromoxynil & neg & 1,19 & $<50$ & 3150 & 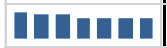 & nd nd nd & -- \\
\hline Candesartan & neg & $-0,3$ & $<350$ & 2653 & 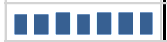 & nd nd nd & nd $\mathrm{nd}$ \\
\hline${ }^{2}$ Carbendazim & neutral & 1,8 & 1 & 7 & 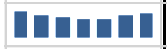 & $-\ldots$ & --- \\
\hline${ }^{2}$ Carbetamid & neutral & 1,65 & $<50$ & 2746 & 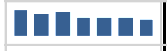 & nd nd nd & -- \\
\hline CBZ & neutral & 2,77 & 540 & 3307 & 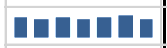 & $-\ldots$ & - nd \\
\hline${ }^{2}$ Chlortoluron & neutral & 2,44 & $<50$ & 2616 & 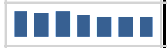 & nd $-\ldots$ & _ nd _ \\
\hline Diatrizoate & neg & $-0,63$ & 104 & 471 & 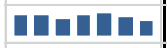 & nd $-\ldots$ & _ nd nd \\
\hline${ }^{2}$ Diuron & neutral & 2,53 & 7 & 1241 & 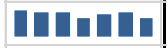 & nd - - & - nd - \\
\hline${ }^{1}$ Fluconazole & neutral & 0,56 & 64 & 617 & 100000| & $-\ldots$ & _ nd _ \\
\hline${ }^{3}$ Hydrochlorothiazide & neutral & $-0,58$ & 24212 & 2770 & 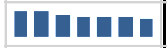 & --- & _ nd _ \\
\hline lopamidol & neutral & $-0,74$ & $<5$ & 45 & 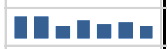 & n.a. & n.a. \\
\hline${ }^{2}$ Isoproturon & neutral & 2,57 & $<50$ & 2908 & 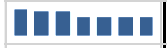 & nd -- & - nd - \\
\hline${ }^{1}$ Lamotrigine & pos & 1,91 & 1068 & 763 & andul| & - nd - & nd nd nd \\
\hline${ }^{2}$ Metoxuron & neutral & 1,77 & $<50$ & 3022 & 100000 & nd - & --- \\
\hline${ }^{2}$ Monuron & neutral & 1,93 & $<50$ & 2895 & 100000 & nd - & _ nd nd \\
\hline Olmesartan & neg & -1 & $<50$ & 3573 & 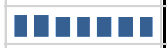 & nd nd _- & nd $n d$ \\
\hline${ }^{1}$ Oxypurinol & neg & 0,23 & 1253 & 902 & 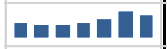 & $-\ldots$ & _ nd nd \\
\hline Primidone & neutral & 1,12 & $<20$ & 95 & 100000 & nd _ nd & n.a. \\
\hline Sucralose & neutral & $-0,47$ & 7584 & 2908 & 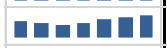 & - nd & _ nd _ \\
\hline${ }^{2}$ Sulfamethazin & neg & 0,21 & $<50$ & 3282 & 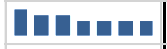 & nd - & - nd - \\
\hline${ }^{1}$ Torasemide & neg & 1,12 & 117 & 695 & 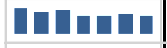 & -- & _ nd nd \\
\hline${ }^{1}$ Xipamide & neg & 1,81 & 12 & 544 & 100000 & $--\ldots$ & _ nd nd \\
\hline \multicolumn{8}{|c|}{ Group 2: easy degradable substances (> $80 \%$ in $\mathrm{B} 01$ and $>30 \%$ in b1) } \\
\hline${ }^{2}$ Atenolol ${ }^{S}$ & pos & $-1,8$ & 1 & 2594 & $\mid------$ & $\square \square \square$ & $\square=\square$ \\
\hline${ }^{1} \mathrm{CBZ}-10-\mathrm{OH}$ & neutral & 1,73 & 345 & 801 & $\boldsymbol{\square}_{------}$ & $-\ldots$ & $\square=$ \\
\hline${ }^{2}$ Citalopram ${ }^{\mathrm{S}}$ & pos & 1,41 & $<350$ & 2989 & $\mid+\ldots$ & $\square \square \square$ & \\
\hline${ }^{1}$ Clarithromycin ${ }^{\mathrm{S}}$ & pos & 2,22 & 9 & 100 & $\mathbf{D}_{------}$ & $\square \square \square$ & $\square \square \square$ \\
\hline${ }^{1}$ Emtricitabine & neutral & $-0,9$ & 143 & 258 & $\mid \mathbf{I}_{-----}$ & nd _ nd & $\square--$ \\
\hline${ }^{1}$ Fexofenadine ${ }^{S}$ & zwitter & 2,93 & 339 & 431 & $\left|\mathbf{D}_{-----}\right|$ & - & $\square \square \square$ \\
\hline${ }^{1}$ Gabapentin & zwitter & $-1,27$ & 1992 & 1489 & $D_{------}$ & nd nd nd & $\square$ nd \\
\hline${ }^{2}$ Ibuprofen & neg & 1,34 & 42 & 251 & $\mathbf{D}_{------}$ & --- & $\square \square-$ \\
\hline${ }^{1}$ lopromide & neutral & $-0,44$ & $<10$ & 101 & $\mid D_{--\ldots--}$ & nd _ nd & $\square=$ \\
\hline${ }^{2}$ Iprovalicarb & neutral & 3,59 & $<50$ & 2297 & $\square_{-----}$ & nd - & $\square--$ \\
\hline${ }^{2}$ Ketoprofen & neg & 0,39 & $<50$ & 1528 & $\boldsymbol{\square}_{------}$ & nd - & $\square \square-$ \\
\hline${ }^{2}$ Levetiracetam & neutral & $-0,59$ & 77 & 2261 & $-\ldots--$ & nd _ - - & $\square \square-$ \\
\hline${ }^{2}$ Metoprolol $^{5}$ & pos & $-0,47$ & 300 & 1298 & $\boldsymbol{D}_{------}$ & - & $\square \square \square$ \\
\hline${ }^{2}$ Naproxen & neg & $-0,05$ & $<200$ & 768 & $\mid D_{------}$ & nd _ nd & $\square \square$ \\
\hline Paracetamol & neutral & 0,9 & $<350$ & 1569 & $\boldsymbol{\square}_{------}$ & nd _ nd & $\square \square \square$ \\
\hline${ }^{2}$ Propachlor & neutral & 2,39 & $<50$ & 1594 & $\|_{-----}$ & nd -- & $\square \square-$ \\
\hline${ }^{1}$ Ramiprilat & neg & $-3,19$ & 265 & 606 & $\mid D_{------}$ & - nd _ - & $\square \square-$ \\
\hline${ }^{2}$ Rufinamid & neutral & 1,27 & $<50$ & 1473 & $\mid \mathbf{I}_{-----}$ & nd - - & $\square \square \square$ \\
\hline${ }^{2}$ Sulfadiazin & neg & $-0,05$ & $<50$ & 3362 & $\mid \mathbf{D}_{-----}$ & nd _ nd & $=-$ \\
\hline${ }^{2}$ Sulfamethoxazol & neg & 0,15 & 208 & 2480 & $\mid \boldsymbol{D}_{------}$ & nd _ _ _ & $\square--$ \\
\hline${ }^{2}$ Sulfapyridin & neg & 0,24 & $<50$ & 3544 & D------ & nd $-\ldots$ & $=--$ \\
\hline${ }^{2}$ Sulfathiazol & neg & 0,5 & $<50$ & 2991 & $\mathbf{I}_{------}$ & nd $-n d$ & $\square=\square$ \\
\hline${ }^{2}$ Trimethoprim ${ }^{\mathrm{s}}$ & pos & 1,1 & 68 & 1493 & $\mid \boldsymbol{D}_{------}$ & - -0 & $\square--$ \\
\hline${ }^{2}$ Trinexapac-ethyl & neg & $-2,51$ & $<50$ & 495 & $\mathbf{D}_{------}$ & nd _ nd & $\square=-$ \\
\hline${ }^{2}$ Valsartan & neg & 0,08 & 2103 & 910 & $\mathbf{D}_{------}$ & $--\ldots$ & $\square \square-$ \\
\hline \multicolumn{8}{|c|}{ Group 3: substances removed all substrate conditions ( $>30 \%$ in $\mathrm{B} 01$} \\
\hline${ }^{2}$ Acetamiprid & neutral & 1,11 & $<50$ & 3460 & $\mathbf{m} \mathbf{m}_{----}$ & nd -- & $-\infty$ \\
\hline Benzotriazole & neutral & 1,29 & 6494 & 6724 & $\| \square m_{----}$ & nd nd nd & $\mathrm{nd}=\mathrm{nd}$ \\
\hline${ }^{2}$ Bezafibrate & neg & 0,74 & 91 & 2290 & $\mid n_{-\ldots--}$ & $-\ldots$ & $\square=$ \\
\hline${ }^{2}$ Capecitabin & neutral & 0,75 & $<50$ & 2870 & $\mid-----$ & nd -- & $\square=$ \\
\hline${ }^{2}$ Clofibrin-Acid & neg & $-0,51$ & $<50$ & 1234 & $\mathbf{m} \mathbf{m}_{----}$ & nd _ nd & $=-$ \\
\hline${ }^{2}$ Deprenyl & neutral & 1,55 & $<50$ & 2201 & $\mid n-\ldots-\ldots$ & nd nd nd & $\square \square=$ \\
\hline${ }^{2}$ Diclofenac & neg & 1,1 & 1388 & 1909 & $\| \square m_{----}$ & nd _ nd & $-\mathrm{nd}$ \\
\hline Diclofenac-4-OH & neg & 0,89 & 221 & 73 & $\mathbf{D}=----$ & n.a. & n.a. \\
\hline${ }^{2}$ Furosemide & neg & $-1,25$ & 256 & 2486 & $\left|\mathbf{m} \mathbf{m}_{----}\right|$ & _ _ nd & $-\mathrm{nd}$ \\
\hline
\end{tabular}




\begin{tabular}{|c|c|c|c|c|c|c|c|}
\hline \\
\hline${ }^{3}$ Mecoprop & neg & $-0,25$ & 27 & 749 & In----- & --- & 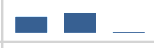 \\
\hline${ }^{2}$ Mianserin & pos & 3,71 & $<350$ & 1193 & In=-ーー- & n.a. & n.a. \\
\hline${ }^{2}$ Pargyline ${ }^{5}$ & pos & 1,41 & $<50$ & 1293 & In-_-_-_ & $=-$ & $\square \square-$ \\
\hline${ }^{2}$ Pheniramine ${ }^{\mathrm{S}}$ & pos & 0,91 & $<500$ & 4265 & Ine_-_- & $-\square \square$ & $-\square \square$ \\
\hline${ }^{2}$ Ranitidin $^{5}$ & pos & 0,45 & $<200$ & 3550 & In----- & nd $\square$ & $\square \square \square$ \\
\hline${ }^{1}$ Sitagliptin ${ }^{5}$ & pos & $-0,14$ & 2163 & 749 & $\mathbf{D} \mathbf{u}_{----}$ & $\square \square \square$ & $-\ldots$ \\
\hline Sotalol & pos & $-2,12$ & $<350$ & 3732 & In=-ー-ー & n.a. & n.a. \\
\hline${ }^{2}$ Venlafaxine ${ }^{S}$ & pos & 1,22 & 409 & 3437 & Ine-_-_- & $\mid \square-\square$ & $-\square \square$ \\
\hline \multicolumn{8}{|c|}{ Group 4: substances with preferred removal at carbon-limited conditions ( $<30 \%$ within $4.2 \mathrm{~d},>30 \%$ overall removal) } \\
\hline${ }^{2}$ Alachlor & neutral & 3,59 & $<200$ & 540 & Un--n- & nd -- & -- \\
\hline${ }^{2}$ Amisulpride ${ }^{\mathrm{S}}$ & pos & 0,09 & 767 & 1228 & 口n_--- & $-\square \square$ & $-\square \square$ \\
\hline${ }^{2}$ Azoxystrobin ${ }^{\mathrm{S}}$ & neutral & 4,22 & $<50$ & 872 & Inn---- & $-\infty=$ & -- \\
\hline${ }^{1} \mathrm{CBZ}-2-\mathrm{OH}$ & neutral & 2,459 & 34 & 647 & Int_--- & --- & --- \\
\hline${ }^{1} \mathrm{CBZ}-3-\mathrm{OH}$ & neutral & 2,46 & 61 & 723 & In_--- & --- & --- \\
\hline${ }^{1}$ Cetirizine $^{\mathrm{S}}$ & neg & 0,65 & 165 & 309 & $\mathbf{D} \mathbf{u}_{----}$ & $-\infty$ & $--\infty$ \\
\hline${ }^{1}$ Clopidogrel-Acid & neg & 1,1 & 163 & 675 & $\mathbf{m} \mathbf{m}_{----}$ & --- & $=-$ \\
\hline${ }^{2}$ Dimefuron & neutral & 3,44 & $<100$ & 2361 & 100menge & nd -- & --- \\
\hline${ }^{2}$ Dimethenamid & neutral & 2,92 & $<50$ & 2315 & Dannen & nd -- & --- \\
\hline${ }^{2}$ Ethofumesat ${ }^{5}$ & neutral & 2,34 & $<100$ & 357 & 10umen- & nd -- & $=-$ \\
\hline${ }^{2}$ Fenhexamid & neg & 4,49 & $<50$ & 129 & 101men_ & nd - nd & -- \\
\hline${ }^{1}$ Flecainide $^{\mathrm{S}}$ & pos & 1,01 & 129 & 308 & $\mathbf{m} \mathbf{m}_{-\ldots-}$ & $-\square$ & $-\square \square$ \\
\hline${ }^{2}$ Flufenacet ${ }^{S}$ & neutral & 3,22 & $<50$ & 718 & In_--- & nd -- & --- \\
\hline${ }^{2}$ Lidocaine $^{5}$ & pos & 2,33 & 102 & 3872 & Ongman & --- & --- \\
\hline${ }^{2}$ Metolachlor ${ }^{5}$ & neutral & 3,45 & $<100$ & 916 & 100men & nd -- & --- \\
\hline Oxazepam & neutral & 2,92 & $<20$ & 35 & IIn-ーーー & & \\
\hline${ }^{1}$ Sulpiride ${ }^{S}$ & pos & $-0,7$ & 84 & 667 & $\mathbf{m} \mathbf{E}_{----}$ & - & $-\infty \square$ \\
\hline${ }^{3}$ Terbuthylazine & neutral & 2,48 & 2 & 174 & 10日meng & -- & - nd \\
\hline${ }^{2}$ Terbutryn & pos & 2,8 & 32 & 51 & Danera & -- & -- \\
\hline Tramadol $^{\mathrm{S}}$ & pos & 0,62 & 182 & 4093 & Ina--- & $-\infty-$ & -- \\
\hline \multicolumn{8}{|c|}{ Group 5: no removal in b1 (>80\% in B01 \& <30\% in b1) } \\
\hline${ }^{2}$ Acesulfame & neg & $-1,49$ & 1771 & 5375 & $\boldsymbol{U}_{------}$ & _ nd _ & nd nd \\
\hline Caffeine & neutral & $-0,55$ & 122 & 1013 & $\mathbf{I}------$ & -- & $\square=$ \\
\hline${ }^{3}$ DEET & neutral & 2,5 & 485 & 1098 & $\mathbf{U}_{------}$ & nd - - & $\square=$ \\
\hline${ }^{1}$ Pregabalin & zwitter & $-1,34$ & 467 & 885 & $\boldsymbol{U}_{------}$ & nd -- & $\square=$ \\
\hline${ }^{2}$ Saccharin & neg & $-0,49$ & 1236 & 753 & 口-ーーーーー & -- & $-\mathrm{nd}$ \\
\hline \multicolumn{8}{|l|}{ Group 6: Other } \\
\hline Diclofenac- $\mathrm{COOH}$ & neg & 2,54 & $<5$ & 43 & - & n.a. & n.a. \\
\hline Saluamine & neg & $-1,91$ & $<100$ & 119 & - $=\mathrm{Gm}$ & n.a. & n.a. \\
\hline Carbanilide & neutral & 3,12 & $<5$ & $<B G$ & & n.a. & n.a. \\
\hline Enalaprilat & neg & $-4,01$ & $<50$ & $<B G$ & & n.a. & n.a. \\
\hline${ }^{2}$ Irgarol & pos & 2,91 & $<100$ & $<B G$ & & nd - - & --- \\
\hline Acyclovir & neutral & $-0,56$ & $<20$ & 34 & ロローーーーー & n.a. & n.a. \\
\hline Ramipril & neg & $-0,18$ & 40 & 12 & 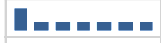 & n.a. & n.a. \\
\hline${ }^{1}$ Azithromycin ${ }^{S}$ & pos & $-1,23$ & $<100$ & 163 & D-anen & $\square \square \square$ & n.a. \\
\hline${ }^{1}$ Climbazole $^{S}$ & pos & 4,3 & 81 & 6 & ㅁㅁ_--- & 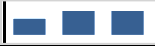 & $\square \square$ \\
\hline
\end{tabular}


S17 Evaluation of plausible initial transformation reaction based on literature data and pathway prediction

\begin{tabular}{|c|c|c|c|c|c|c|c|}
\hline \multirow{2}{*}{ compound } & \multirow{2}{*}{ group } & \multirow{2}{*}{$\begin{array}{l}\text { PPS rule (bold reactions confirmed by } \\
\text { literature data) }\end{array}$} & \multirow{2}{*}{ reactions from literature } & \multirow{2}{*}{ literature } & \multirow{2}{*}{$\begin{array}{l}\text { transformation } \\
\text { product }\end{array}$} & \multirow{2}{*}{$\begin{array}{c}\text { column } \\
\text { experiment }\end{array}$} & batch experiment \\
\hline & & & & & & & B01 b1 \\
\hline racetamol & & 2 bt0067 & hydrolysis of secondary amide (anilide) & doi.org/10.1021/es101035b & & & \\
\hline uprofen & & 2 bt0051;bt0241;bt0242;bt0333 & hydroxylation of alkyl chain & doi.org/10.1016/j.watres.2005.04.068 & & & \\
\hline & & & & doi.org/10.1016/j.watres.2005.04.068; & & & \\
\hline aproxen & & 2 bt0023;bt0241 & O-dealkylation (O-demethylation) & $\begin{array}{l}\text { doi.org/10.1007/s00244-010-9622-2; } \\
\text { doi.org/10.1016/j.watres.2018.03.040 }\end{array}$ & & & \\
\hline vetiracetam & & 2 bt0027;bt0242;bt0243;bt0334 & primary amide hydrolysis & doi.org/10.1007/s00244-010-9622-2 & & & \\
\hline finamid & & 2 bt0027 & primary amide hydrolysis & doi.org/10.1021/acs.est.8b02763 & & & \\
\hline miprilat & & 2 bt0051;bt0063;bt0241 & & & & & \\
\hline Ifathiazol & & 2 bt0144 & pterin-conjugation & doi: 10.1021/acs.est.7b06716 & & & \\
\hline inexapac-ethyl & & 2 bt0024;bt0044;bt0071 & ester hydrolysis & doi.org/10.1021/acs.est.8b02763 & & & \\
\hline Isartan & & 2 bt0051;bt0241;bt0242;bt0243;bt0334 & $\mathrm{N}$-dealkylation & doi.org/10.1021/es101035b & Valsartanic-Acid & 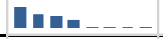 & 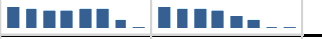 \\
\hline abapentin & & 2 bt0063 & $\mathrm{N}$-dealkylation & doi.org/10.1016/j.watres.2018.01.027 & Gabapentin-Lactam & $\| \mathrm{m}=---\_$ & 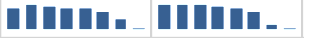 \\
\hline rovalicarb & & 2 bt0067;bt0318 & hydroxylation of aromatic methyl & doi.org/10.1021/acs.est.8b02763 & & & \\
\hline etoprofen & & 2 bt0353 & hydroxylation of aromatic ring & doi.org/10.1016/j.watres.2005.04.068 & & & \\
\hline $3 \mathrm{Z}-10-\mathrm{OH}$ & & 2 bt0002;bt0068 & oxidation of secondary alcohol (dehydrogenation) & doi.org/10.1021/es5024493 & & & \\
\hline promide & & 2 bt0001;bt0002;bt0023;bt0067;bt0243 & oxidation of primary alcohol (dehydrogenation) & doi.org/10.1021/es800789r & & & \\
\hline ntricitabine & & 2 bt0001;bt0162;bt0243;bt0259 & oxidation of primary alcohol (dehydrogenation) & doi.org/10.1016/j.watres.2016.03.045 & Emtricitabine- $\mathrm{COOH}$ & ann_--n_ & 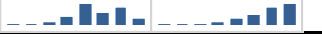 \\
\hline Ifamethoxazol & & 2 bt0144 & pterin-conjugation & doi: 10.1021/acs.est.7b06716 & & & \\
\hline Ifadiazin & & 2 bt0144 & pterin-conjugation & doi: 10.1021/acs.est.7b06716 & & & \\
\hline Alfapyridin & & 2 bt0005;bt0144 & pterin-conjugation & doi: 10.1021/acs.est.7b06716 & & & \\
\hline ecoprop & & 3 bt0023;bt0036 & O-dealkylation & doi.org/10.1016/S0169-7722(02)00206-1 & & & \\
\hline pecitabin & & 3 bt0002;bt0318 & hydroxylation of alkyl chain, carbamate hydrolysis & doi.org/10.1021/acs.est.8b02763 & & & \\
\hline ezafibrate & & 3 bt0051;bt0067;bt0243 & secondary amide hydrolysis & doi.org/10.1021/es101035b & & & \\
\hline enzotriazole & O & 3 bt0005 & methylation, hydroxylation of aromatic ring & doi.org/10.1021/es405694z & & & \\
\hline Irosemide & & 3 bt0063;bt0144;bt0359;bt0375 & $\mathrm{N}$-dealkylation & doi.org/10.1016/j.aca.2013.12.012 & & & \\
\hline ofibrin-Acid & & 3 bt0005;bt0051 & hydroxylation of alkyl chain & doi.org/10.1016/j.jhydrol.2009.04.006 & & & \\
\hline clofenac & 0 & 3 bt0065 & $\begin{array}{l}\text { amidation, hydroxylation of aromatic ring, } \\
\text { decarboxylation, oxidation }\end{array}$ & doi.org/10.1016/j.watres.2016.08.002 & Diclofenac- $\mathrm{COOH}$ & 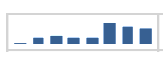 & $-\ldots--\infty \mathbf{U}_{-}$ \\
\hline etamiprid & 0 & 3 bt0028;bt0350 & nitrile hydrolysis & doi.org/10.1021/acs.est.8b02763 & & & \\
\hline opidogrel-Acid & O & 4 bt0005;bt0051;bt0063;bt0162 & & & & & \\
\hline achlor & O & 4 bt0022;bt0023;bt0242;bt0243 & Cl-cleavage (substitutive with GSH), reductive dehalogen & a doi.org/10.1021/acs.est.8b02763 & & & \\
\hline rbutryn & O & 4 bt0330;bt0339 & S-oxidation & doi.org/10.1021/es403531d & Terbutryn-S-O & 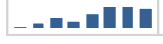 & - \\
\hline $3 \mathrm{Z}-3-\mathrm{OH}$ & $\bigcirc$ & 4 bt0068 & one electron transfer (formation of phenoxy radicals) & doi.org/10.1021/acs.est.5b02737 & & & \\
\hline nhexamid & 0 & 4 bt0067 & & & & & \\
\hline $3 \mathrm{Z}-2-\mathrm{OH}$ & 0 & 4 bt0068 & $\begin{array}{l}\text { nitration, oxidation with simultaneous loss of carbamoyl } \\
\text { group }\end{array}$ & doi.org/10.1021/acs.est.5b02737 & & & \\
\hline ufenacet & 0 & 4 bt0023;bt0065;bt0162;bt0243 & ether cleavage (substitutive with GSH) & doi.org/10.1021/acs.est.8b02763 & & & \\
\hline etolachlor & O & 4 bt0022;bt0023;bt0036;bt0242;bt0243 & $\begin{array}{l}\text { Cl-cleavage (substitutive with GSH), reductive } \\
\text { dehalogenation }\end{array}$ & $\begin{array}{l}\text { doi.org/10.1021/es990686z; } \\
\text { doi.org/10.1021/es9503600 }\end{array}$ & & & \\
\hline erbuthylazine & 0 & 4 bt0330;bt0339 & $\mathrm{N}$-dealkylation, hydrolytic dechlorination & doi.org/10.2134/jeq2003.1089 & _Terbuthylazine-2-OH & & 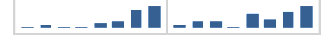 \\
\hline $\begin{array}{l}\text { imethenamid } \\
\text { imefuron }\end{array}$ & & $\begin{array}{l}4 \text { bt0022;bt0023;bt0036;bt0162;bt0243 } \\
4 \text { bt0068;bt0243 }\end{array}$ & Cl-cleavage (substitutive with GSH), reductive dehalogen & a doi.org/10.1021/acs.est.8b02763 & & & \\
\hline esulfame & & 5 bt0144 & hydrolysis of sulfonic acid ester & doi.org/10.1016/j.watres.2016.11.041 & & & \\
\hline ET & & 5 bt0036;bt0243 & $\mathrm{N}$-dealkylation & doi.org/10.1021/es101035b & & & \\
\hline egabalin & & 5 bt0063;bt0241 & amidation, $\mathrm{N}$-dealkylation & Henning et al (submitted) & & & \\
\hline ffeine & & 5 bt0063;bt0243;bt0316 & N-dealkylation & doi.org/10.1007/s10529-006-9196-2 & & & \\
\hline
\end{tabular}

